\title{
3D printing of long period gratings for curvature applications
}

\author{
Nuno F. Valente, Lúcia Bilro, and Ricardo Oliveira* \\ Instituto de Telecomunicações and Universidade de Aveiro, Campus Universitário de Santiago, 3810-193 Aveiro, Portugal
}

\begin{abstract}
In this paper we will discuss a new type of long period grating (LPG) that consists on an optical fibre glued on top of a 3D printed grooved plate. The LPG performance will be tested for the case when pressure is applied transversely to the grooved plate. Results showed the appearance of attenuation bands at specific wavelengths, that grow with increasing load. The evolution of the dip power at those resonances, as function of the curvature will be shown, demonstrating the potentialities of the sensor for low cost intensity detection schemes.
\end{abstract}

\section{Introduction}

Nowadays the possibility to change the guiding properties of optical fibres is being used in both sensor and communication applications. One way to achieve this effect is by using short and long period gratings (LPGs). These are structures capable to periodically modulate the refractive index of the fibre core. An LPG has typically a period between $100 \mu \mathrm{m}$ and $1 \mathrm{~mm}$. In this structure the fundamental core mode is coupled to certain cladding modes, causing attenuation bands to appear in the transmission spectrum at certain wavelengths defined by:

$$
\lambda_{i}=\left(n_{e}-n_{i}\right) \Lambda
$$

where $\lambda_{i}$ is the wavelength of the resonance band, $n_{e}$ is the effective refractive index of the core mode, $n_{i}$ is the refractive index of mode $i^{\text {th }}$ cladding mode, and $\Lambda$ is the grating period. The light transmission at the resonances could be expressed by the following expression [1]:

$$
T=\cos ^{2}\left(k_{c o-c l}^{a c} l\right)
$$

where $l$ is the length of the grating and $k_{c o-c l}^{a c}$ is the $a c$ component of the coupling coefficient between the core and the cladding modes.

In literature it is possible to find several types of LPGs and two major groups stand out: permanent gratings through laser engraving and mechanically induced through load. For the first, there are several methods to produce them, such as $\mathrm{CO}_{2}$ laser [2], femtosecond laser [3], ion beam [4], and electric arc discharge [5]. For the later, there are also different procedures to fabricate them, such as: pressing a grooved plate $[6,7]$ or laminated plate [8] onto an optical fibre, and recently, by surface bonding an optical fibre to a grooved plate, namely by torsion and shear-strain forces [9]. In this type of gratings, the refractive index of the fibre core is modulated according to the force applied and may be expressed by [9]:

$$
\Delta n=-n_{0} p_{e} \varepsilon_{i}=-n_{0} p_{e} \frac{F}{E_{i} A_{i}}
$$

being, $n_{0}, p_{e}, \varepsilon_{\mathrm{i}}$ and $F$, the unperturbed refractive index, the effective photoelastic constant, the associated strain and the applied force, respectively. $E$ and $A$ are the effective Young's Modulus and radius of each region, respectively.

Taking this later example, in this work it will be demonstrated the capability to measure curvature through these fibre optic devices.

\section{Methods}

\subsection{Sensor fabrication}

The fabrication of the sensor followed the descriptions found in [9]. For that, the grooved plate was 3D printed using a digital light projector (DLP) 3D printer from Anycubic[10]. The plate has a total of 100 rectangular hollow regions, separated with a period of $650 \mu \mathrm{m}$, giving a total grooved plate length of $65 \mathrm{~mm}$. The thickness of the plate was $5 \mathrm{~mm}$ and its width was $15 \mathrm{~mm}$. The resin used for the 3D printing was a standard ultraviolet (UV) transparent resin from Anycubic. The computer aided design (CAD) image of the proposed grooved plate may be seen in Fig. 1(a).

The assembly of the fibre structure is done by positioning the optical fibre on top of the grooved plate followed by surface bonding it using the same UV resin that composes the grooved plate. When performing this step, some care must be taken, such as not placing too much glue between the optical fibre and the grooved plate, otherwise it may fill the hollow regions of the plate, taking away its periodicity. Another precaution that must be taken when performing this task is to keep the optical fibre completely stretched during the adhesion process, otherwise it may not be well fixed to the grooved plate. Once this step is completed, the fibre structure is ready to be mechanically characterized in order to induce the LPG.

\footnotetext{
* Corresponding author: oliveiraricas@av.it.pt
} 


\subsection{Curvature characterization}

The mechanical apparatus used for the curvature characterization may be seen on Fig. 1 b) and c).

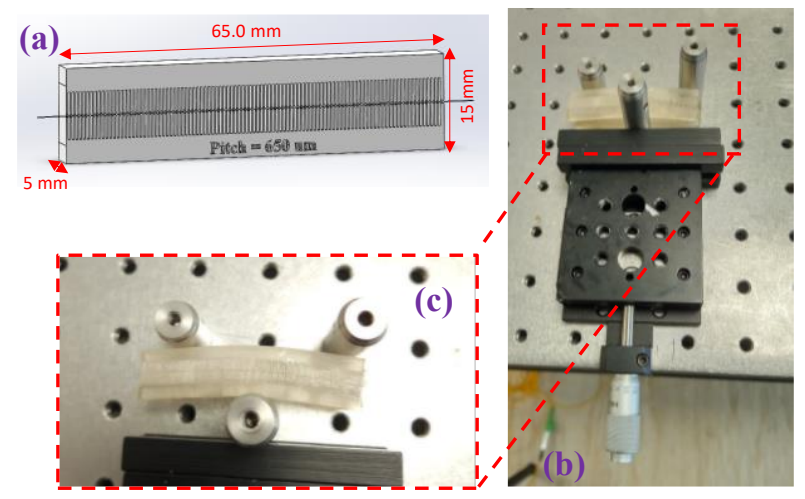

Fig. 1. (a) CAD image of the grooved plate. (b) Experimental setup for the curvature test. (c) Inset of the sensor under curvature.

The characterization setup is based on two mechanical posts tightened to an optical table, allowing to secure the fibre device at both ends. Then, a transversal force is applied at the middle region of the grooved plate, using a micrometer screw (10 $\mu$ m resolution). The displacement was made in steps of $50 \mu \mathrm{m}$ up to $300 \mu \mathrm{m}$. The curvature was calculated through the following expression [11]:

$$
C=\frac{1}{R}=\frac{2 h}{h^{2}+D^{2}}
$$

where $C$ is the curvature, $R$ is the radius of curvature, $h$ is the displacement imposed by the micrometer screw and $D$ is the distance between the fixed posts $(50.7 \mathrm{~mm})$. Considering the values given for $h$ and $D$, the curvature was made in steps of $0.04 \mathrm{~m}^{-1}$ up to a maximum value of $0.23 \mathrm{~m}^{-1}$.

The curvature characterization was made in transmission, using a wideband light source (Fianium Whitelease model SC-400-2) and an optical spectrum analyzer (Advantest Q8384), is used to collect the grating spectra after each curvature step.

In order to see the influence of the uniaxial strain, we decide to measure the polarization dependent losses (PDL) of the fibre device. To accomplish this task, we used an optical network analyzer (Agilent Technologies 86038b).

\section{Results}

The spectra obtained for the different steps of curvature may be seen in Fig. 2.

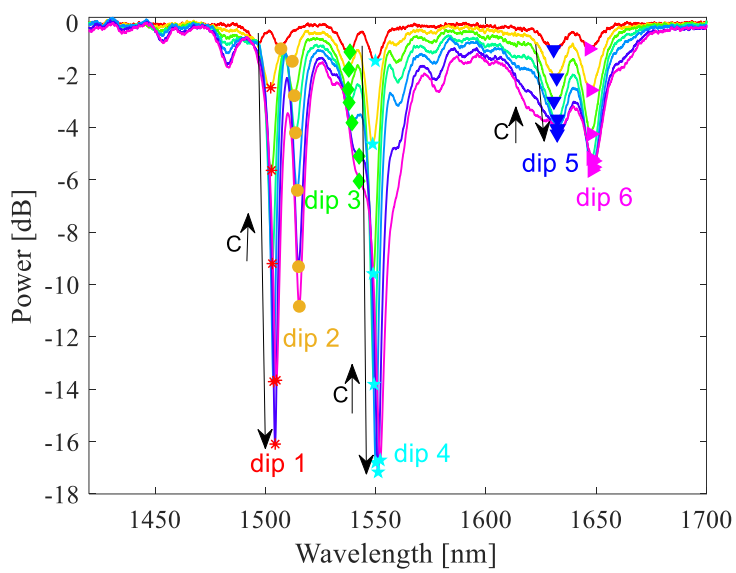

Fig. 2. Transmission spectra as function of the applied curvature.

The results presented on Fig. 2 show the appearance of six dip resonances that increase their attenuation with the increase of curvature value. By tracking the dip attenuation of the resonances that appear in Fig. 2 we were able to represent the evolution of the coupling strength as function of the applied curvature, as is shown in Fig. 3.

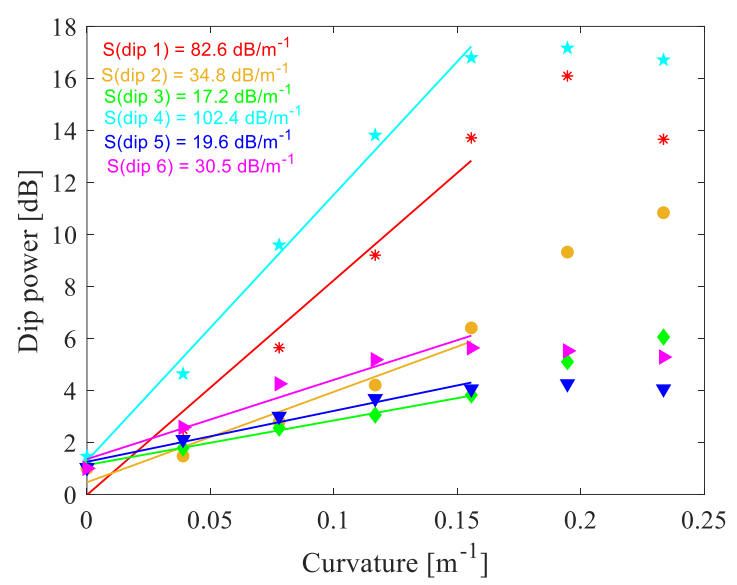

Fig. 3. Dip power of the resonance bands that appear in Fig. 2, as function of the applied curvature.

From Fig. 3, it can be seen that the dip resonances follow a linear relationship with the applied curvature up to 0.15 to $0.20 \mathrm{~m}^{-1}$. Then, the coupling strength from the core mode to the cladding mode starts to be less intense, and later, they reach a maximum value, as can be observed for instance for the $1^{\text {st }}$ and $4^{\text {th }}$ dip resonances. At this point, $\sim 0.2 \mathrm{~m}^{-1}$, the attenuation bands reached bandwidths $\leq 4 \mathrm{~nm}$ and relatively small background losses $(<1 \mathrm{~dB})$. After reaching the minimum attenuation value, $\sim 16 \mathrm{~dB}$ to $\sim 17 \mathrm{~dB}$, respectively for the $1^{\text {st }}$ and $4^{\text {th }}$ dip resonances, the transmission starts to increase again, where the coupling is now reversed, being the cladding modes coupled to the core mode. The process continues up to maximum transmission (minimum attenuation). However, we stopped the process in order to prevent plastic deformation of the polymeric structure. Nevertheless, it is expected that the attenuation bands present an oscillation between maxima and a minima values, as indicated by the cosine function found in equation (2). In Fig. 3 it is also possible to observe that the sensitivities of the attenuation 
bands as function of curvature are $82.6 \mathrm{~dB} / \mathrm{m}^{-1}$, $34.8 \mathrm{~dB} / \mathrm{m}^{-1}, 17.2 \mathrm{~dB} / \mathrm{m}^{-1}, 102.4 \mathrm{~dB} / \mathrm{m}^{-1}, 19.6 \mathrm{~dB} / \mathrm{m}^{-1}$ and $30.5 \mathrm{~dB} / \mathrm{m}^{-1}$ for the $1^{\text {st }}, 2^{\text {nd }}, 3^{\text {rd }}, 4^{\text {th }}, 5^{\text {th }}$ and $6^{\text {th }}$ dip resonances, respectively. These values were slightly lower than those found in literature (that is about $130.4 \mathrm{~dB} / \mathrm{m}^{-1}$ ) for other fibre optic structure based on multimode interference [12]. Despite this, we stress out that the sensitivities are still high compared to the state of the art and that the fabrication methodology used in this work is simpler and cost effective. Furthermore, we observed that the resonance bands have a wavelength shift of less than $1.5 \mathrm{~nm}$ during the linear tuning range. This low value could be an interesting opportunity to build a fibre device operating with low cost approaches, using a laser source cantered at one of the resonance bands and using a photodetector to monitor the power during time [9].

In order to compare the results obtained in Fig. 2 with the ones predicted theoretically, we decide to run a simulation using the OptiGrating v4.2 software (Optiwave Corporation). The simulations were performed considering the first three linearly polarized modes (LP modes), an estimated refractive index of 1.44912 and 1.44400 , and diameters of $8.2 \mu \mathrm{m}$ and $125.0 \mu \mathrm{m}$, for the core and cladding, respectively. The grating period was set to $650 \mu \mathrm{m}$ and the refractive index modulation was optimised in order to fit the spectral profile obtained experimentally. The results of this simulation, together with the ones obtained experimentally for a curvature of $0.19 \mathrm{~m}^{-1}$ are shown in Fig. 4

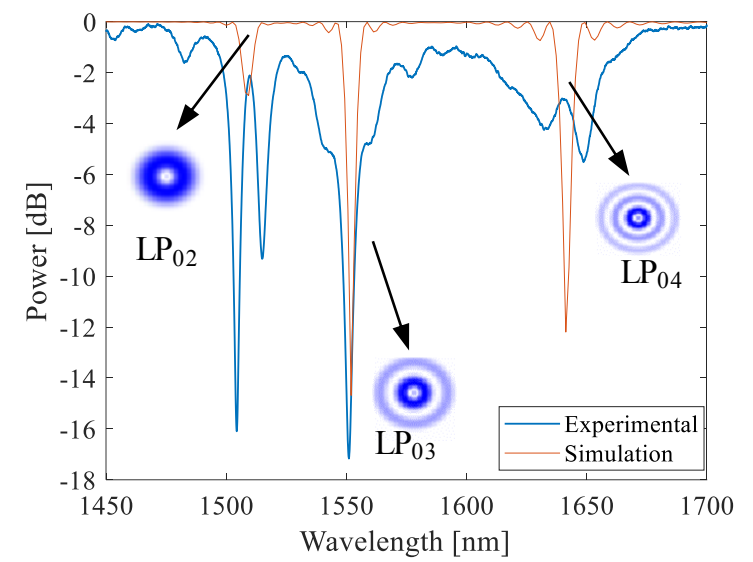

Fig. 4. Experimental and theoretical spectrum results. The images indicate the corresponding modes for which the coupling has occured.

As is seen in Fig. 4, the simulation results revealed three dip resoance bands located at $\sim 1497 \mathrm{~nm}, \sim 1540 \mathrm{~nm}$ and $\sim 1628 \mathrm{~nm}$, that corresponde to the $\mathrm{LP}_{02}, \mathrm{LP}_{03}$ and $\mathrm{LP}_{04}$ modes, respectively. Yet, each of these resonaces are split into two on the experimental results. However, we stress out that the result observed experimentaly is an indicative of the different refractive index modulation observed in both orthogonal polarizations. To deeply explore this effect we decide to measure the PDL of the fibre device. The results may be seen in Fig.

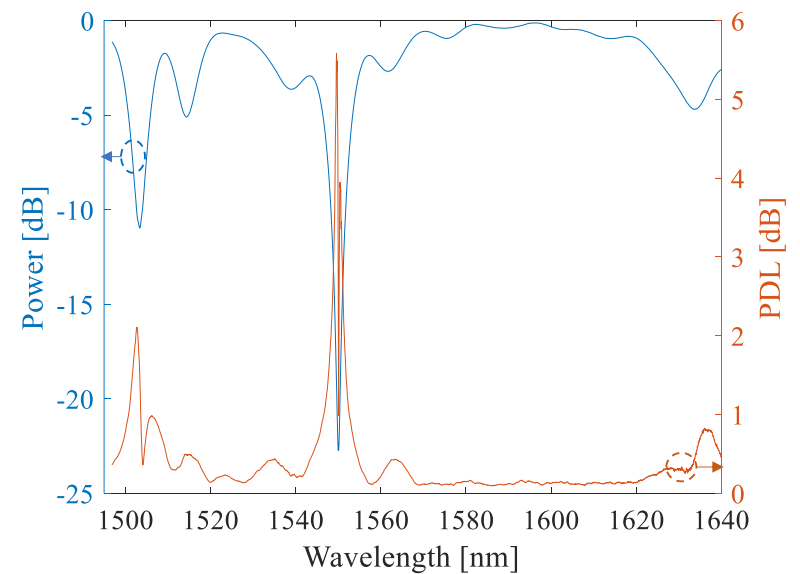

Fig. 5. PDL and insertion loss of the fibre device.

In the graph of 5 it is possible to observe the existence of a high PDL value, reaching values in the order of $\sim 5.6 \mathrm{~dB}$. This happens because asymmetric stresses are applied to the optical fibre bonded to the grooved plate, creating different refractive index variations in the two orthogonal axes of the fibre, which leads to the appearance of two attenuation bands in the transmission spectrum. This difference in modulation of the refractive index is due to the geometry of the fibre device, because, the fibre is surface bonded to the grooved plate and this restricts the fibre core to be positioned at $2.563 \mathrm{~mm}$ out of the central region (neutral region), considering half of the thickness of the grooved plate and half of the diameter of the optical fibre. Note that if the fibre is exactly at the middle region of the grooved plate, no strain would be induced at the core of the optical fibre. Because of that, an asymmetric strain distribution is created when the fibre is being curved, being one side of the fibre compressed while the other is being elongated. As a result, different refractive index modulations are built and thus, different coupling strengths could be found for the two orthogonal polarizations. This effect could in fact be detrimental in different applications and to solve this issue we propose the use of a polariser.

\section{Conclusion}

In this work, a low-cost LPG curvature sensor is proposed. The spectral results showed attenuation bands up to $17.2 \mathrm{~dB}$, located at specific wavelengths that satisfy the resonance condition, presenting also low losses outside of the resonance bands $(1.2 \mathrm{~dB})$. The results revealed that the dip attenuation bands reach a linear sensitivity with increasing curvature, reaching values up to $\sim 102.4 \mathrm{~dB} / \mathrm{m}^{-1}$, which is a high value compared to the state of the art. Besides that, the low cost and simplicity of the proposed fabrication process, makes this fibre sensor well suited for curvature sensing applications. Furthermore, it is worth to mention that the device could be used with simple detection schemes such as the ones based in intensity, by using a laser centred at one of the resonance bands and monitoring the power variation through a photodetector, allowing to reduce the cost and the complexity of the final device. 
This work was funded by FCT-Fundação para a Ciência e Tecnologia and by the European Regional Development Fund (FEDER), through the Competitiveness and Internationalization Operational Programme (COMPETE 2020) of the Portugal 2020 framework and by national funds under the projects FOPEComSens (PTDC/EEI-TEL/1511/20), AQUATICsens (POCI-01-0145-FEDER-032057) and UIDB/50008/2020UIDP/50008/2020.

\section{References}

[1] T. Erdogan, "Fiber grating spectra," J. Light. Technol., vol. 15, no. 8, pp. 1277-1294, 1997, doi: 10.1109/50.618322.

[2] J. M. P. Coelho, C. Silva, M. Nespereira, M. Abreu, and J. Rebordão, "Writing of Long Period Fiber Gratings Using CO2 Laser Radiation," $A d v$. Opt. Fiber Technol. Fundam. Opt. Phenom. Appl., vol. 32, pp. 287-314, Feb. 2015, doi: $10.5772 / 59153$.

[3] B. Li, L. Jiang, S. Wang, H. L. Tsai, and H. Xiao, "Femtosecond laser fabrication of long period fiber gratings and applications in refractive index sensing," Opt. Laser Technol., vol. 43, no. 8, pp. 1420-1423, 2011, doi: 10.1016/j.optlastec.2011.04.011.

[4] M. L. von Bibra, A. Roberts, and J. Canning, "Fabrication of long-period fiber gratings by use of focused ion-beam irradiation," Opt. Lett., vol. 26, no. 11, pp. 765-767, 2001, doi: 10.1364/ol.26.000765

[5] C. Colaço, P. Caldas, I. Del Villar, R. Chibante, and G. Rego, "Arc-induced long-period fiber gratings in the dispersion turning points," J. Light. Technol., vol. 34, no. 19, pp. 4584-4590, 2016, doi: 10.1109/JLT.2016.2540678.
[6] R. Oliveira, L. M. Sousa, A. M. Rocha, R. Nogueira, and L. Bilro, "UV inscription and pressure induced long-period gratings through 3D printed amplitude masks," Sensors, vol. 21, no. 6, 2021, doi: 10.3390/s21061977.

[7] S. H. Shi, X. J. Zhou, Z. Y. Zhang, L. Lan, C. Yin, and Y. Liu, "Fiber-optic solution concentration sensor based on a pressure-induced long-period grating in a composite waveguide," Chinese Phys. Lett., vol. 29, no. 9, 2012, doi: 10.1088/0256307X/29/9/094206.

[8] I. Torres-Gómez, D. E. Ceballos-Herrera, and K. M. Salas-Alcantara, "Mechanically-induced long-period fiber gratings using laminated plates," Sensors, vol. 20, no. 9, 2020, doi: $10.3390 / \mathrm{s} 20092582$.

[9] R. Oliveira, R. Nogueira, and L. Bilro, "3D printed long period gratings and their applications as high sensitivity shear-strain and torsion sensors," Opt. Express, vol. 29, no. 12, pp. 17795-17814, 2021, doi: 10.1364/oe.427387.

[10] "ANYCUBIC 3D Printing." https://www.anycubic.com/ (accessed Jul. 30, 2021).

[11] Y. Liu, L. Zhang, J. A. R. Williams, and I. Bennion, "Optical Bend Sensor Based on Measurement of Resonance Mode Splitting of Long-Period Fiber Grating," IEEE Photonics Technol. Lett., vol. 12, no. 5, pp. 531-533, 2000, doi: 10.1109/68.841276.

[12] Y. Gong, T. Zhao, Y. J. Rao, and Y. Wu, "Allfiber curvature sensor based on multimode interference," IEEE Photonics Technol. Lett., vol. 23, no. 11, pp. 679-681, 2011, doi: 10.1109/LPT.2011.2123086. 